\title{
AN AUTOMATIC SEGMENTATION METHOD FOR SCANNED IMAGES OF WHEAT ROOT SYSTEMS WITH DARK DISCOLOURATIONS
}

\author{
JAROSŁAW GOCŁAWSKI*, JOANNA SEKULSKA-NALEWAJKO *, EWA GAJEWSKA **, \\ MARZENA WIELANEK ** \\ * Computer Engineering Department \\ Technical University of Łódź, Stefanowskiego 18/22, 90-924 Łódź, Poland \\ e-mail: \{jgoclaw, jsekulska\}@kis.p.lodz.pl \\ ${ }^{* *}$ Department of Plant Physiology and Biochemistry \\ University of Łódź, Banacha 12/16, 90-237 Łódź, Poland \\ e-mail: \{ewagaj, mawie\}@biol.uni.lodz.pl
}

\begin{abstract}
The analysis of plant root system images plays an important role in the diagnosis of plant health state, the detection of possible diseases and growth distortions. This paper describes an initial stage of automatic analysis - the segmentation method for scanned images of Ni-treated wheat roots from hydroponic culture. The main roots of a wheat fibrous system are placed separately in the scanner view area on a high chroma background (blue or red). The first stage of the method includes the transformation of a scanned RGB image into the HCI (Hue-Chroma-Intensity) colour space and then local thresholding of the chroma component to extract a binary root image. Possible chromatic discolourations, different from background colour, are added to the roots from blue or red chroma subcomponent images after thresholding. At the second stage, dark discolourations are extracted by local fuzzy c-means clustering of an HCI intensity image within the binary root mask. Fuzzy clustering is applied in local windows around the series of sample points on roots medial axes (skeleton). The performance of the proposed method is compared with hand-labelled segmentation for a series of several root systems.
\end{abstract}

Keywords: root system image, segmentation, skeleton, root discolourations, fuzzy c-means clustering.

\section{Introduction}

The plant root systems analysis is very important in biological research (Zobel et al., 2007; Smit et al., 2000). It provides quick, coarse information about the health state of the plants, the presence of possible diseases or growth distortions. It can also be helpful in the evaluation of plant tolerance to the destructive influence of different chemical agents such as heavy metals (Terry et al., 2000; Jung et al., 2004; Seregin and Kozhevnikova, 2009). Plant roots respond to heavy metal stress not only with the restriction of growth, but also with the appearance of toxicity symptoms such as discolourations, usually greyish or brownish. Additionally, after histochemical or vital staining, blue, yellow or red discolourations can be observed (cf. Figs. 1 and 2). Root image segmentation is the process of dividing the image into regions of interest, usually root objects and a background (Smit et al., 2000). In the case considered also discolouration regions are to be separated from roots and the background.
Despite the large number of segmentation methods using thresholding, edge filtering, watersheds, textures, region growing (Gonzalez and Woods, 2008; Gonzalez et al., 2004; Vincent and Soille, 1991), no general segmentation method exists. Practically almost every class of images needs an individual approach. In a traditional approach, washed roots are properly stained to ensure good contrast with the background in grey-level scanner or camera images (Smit et al., 2000; Glasbey and Horgan, 1995). Then global thresholding methods can establish which levels belong to root objects. Automatic thresholding is based on searching for the minimum between two peaks of a bimodal image histogram. For unstained white roots, a dark background is selected to enhance the image contrast (Smit et al., 2000).

A lot of plant diseases, including those resulting from environmental pollution, are often manifested in root systems as different colour nonuniformities. Discolourations on root surfaces can be effectively segmented and anal- 


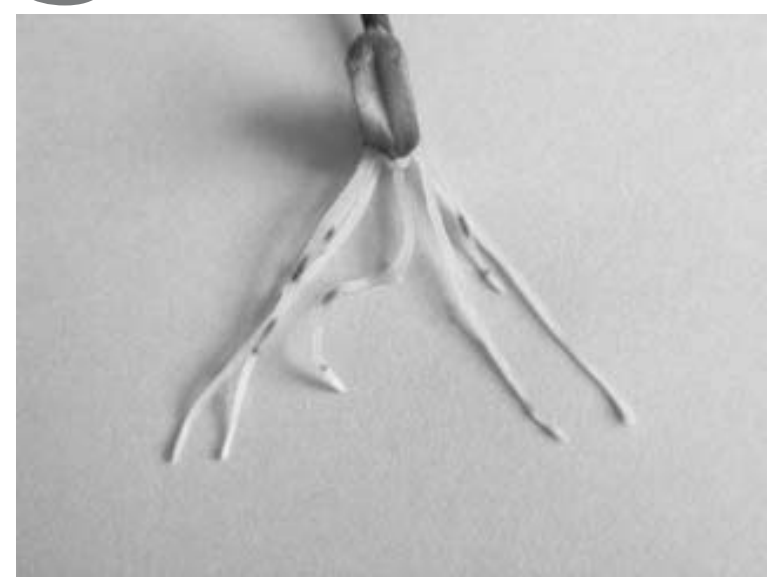

Fig. 1. Exemplary photo of an Ni-treated wheat root system with coloured areas (dark places, originally red) after staining for lignin detection.

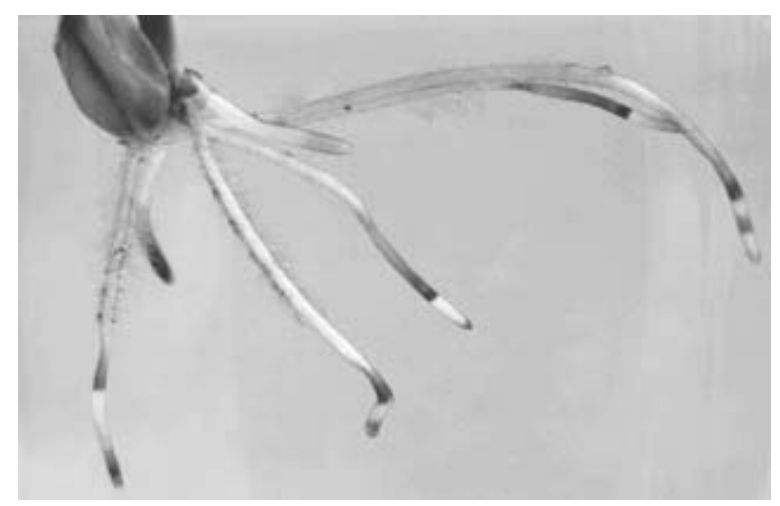

Fig. 2. Exemplary photo of an Ni-treated wheat root system with coloured areas (dark places, originally blue) after staining with Evans blue for dead cells detection.

ysed only in colour images. Commercial programs like WinRHIZO (Regent Instruments Inc., 2008) include basic methods to define colour classes, which can be grouped around selected colour hues. Similar, elementary colour analyses can be carried out using Photoshop or GIMP (Nord, 2008). When certain type discolourations must be detected in the roots, which have nonuniform, variable brightness, special methods should be developed.

\section{Root image segmentation preliminaries}

In this paper, images of wheat seedlings population from hydroponic culture are taken into account. Hydroponically grown, healthy roots are of white colour. In the tested population of wheat roots, the authors observed local achromatic darkenings with variable intensity. A computer measurement system has been completed at the Department of Plant Physiology and Biochemistry of the University of Łódź for the purpose of root systems analysis including the segmentation of root images with dis- colourations. It consists of a standard scanner device, with 300/600 dpi and 24 bit/pixel resolutions, connected to a PC computer with a Windows Vista operating system and a MATLAB environment. Scanner images, acquired as JPEG files, are subjects of segmentation in the MATLAB programming environment. The wheat belongs to plants with a fibrous root system. To minimize the overlapping of roots, in the zone around the common origin, they are initially separated and laid out vertically, along the horizontal direction in front of the scanner glass (Fig. 47). The image background is made of a plastic sheet with colour approximating pure red or blue RGB components to get a maximal hue distance from the expected chromatic discolourations. The scanner scanner device applied ensures fully repetitive image acquisition conditions. It has been verified that scanned images of root systems are always provided with very uniform illumination. Moreover, chroma images from the HCI space to be thresholded are independent of any hypothetical illumination changes because of their differential nature. The authors assume that images can be scanned with 300 dpi or 600 dpi horizontal resolutions (only the first case has been verified). To preserve equal terms of a program running at $600 \mathrm{dpi}$, all suggested pixel values of algorithm parameters in the $X Y$ plane should be doubled. The proposed method consists of the steps described in the subsections below. The images can represent only two-dimensional views of three-dimensional root system objects. Nevertheless, statistical parameters describing the rate of discolourations appearance should be preserved.

\section{Hybrid method of root image segmentation}

A new method of root image segmentation has been proposed, based on the observation that healthy root tissue as well as the searched darkening regions are mostly achromatic. In the examined root images, achromatic areas of interest usually extend over the wide range of brightness, from black to white, and sometimes coexist with highly chromatic discolouration regions.

3.1. RGB to HCI colour transformation. Colour images loaded into the MATLAB workspace have the classical Red-Green-Blue (RGB) representation (The Mathworks Inc., 2008a). To separate the achromatic root components from a highly chromatic background (e.g., blue), the image chroma component should be found. One of the possible solutions is the transformation of the RGB into the HSV colour space and the extraction of the saturation image $S$ (Gonzalez et al., 2004). The authors adopted the HCI (Hue-Chroma-Intensity) colour space (Lambert and Carron, 1999). This selection ensures that equal distances in the tristimulus space correspond to equal perceptual differences between colours, and it enables the minimization 
of nonuniform noise sensitivity accompanying nonlinear transforms. Besides, more than one resultant image component can be useful in the segmentation process. The HCI space is defined as the combination of:

- a linear transform of RGB to $Y C_{1} C_{2}$ components:

$$
\left[\begin{array}{l}
Y \\
C_{1} \\
C_{2}
\end{array}\right]=\mathbb{T}_{Y C} \times\left[\begin{array}{c}
R \\
G \\
B
\end{array}\right],
$$

with the transform matrix

$$
\mathbb{T}_{Y C}=\left[\begin{array}{crr}
1 / 3 & 1 / 3 & 1 / 3 \\
1 & -1 / 2 & -1 / 2 \\
0 & -\sqrt{3} / 2 & \sqrt{3} / 2
\end{array}\right],
$$

- a nonlinear transform providing HCI components:

$$
\begin{gathered}
I=Y, \\
C=\sqrt{C_{1}^{2}+C_{2}^{2},} \\
H= \begin{cases}\arccos \left(C_{1} / C\right) & \text { if } C_{1}>0, \\
2 \pi-\arccos \left(C_{1} / C\right) & \text { otherwise. }\end{cases}
\end{gathered}
$$

Arrays of $C(x, y)$ and $I(x, y)$ for each pixel $(x, y)$ represent grey level chroma and intensity images denoted as $\mathcal{I}_{C}$ and $\mathcal{I}_{I}$, respectively. The hue component formally describing the colour itself as an angle $\left[0-360^{\circ}\right]$ is useless due to its high sensitivity to noise at low saturations (chroma). High saturation root colourations observed in our experiments were "red-like" type, which includes tones of red, brown and yellow. The colour space transform presented in Eqn. (1) outputs two chroma components, $C_{1}$ and $C_{2}$, of red and blue primaries, respectively (Fig. 3).

3.2. Thresholding of component images. The complex chroma image $\mathcal{I}_{C}$ must be thresholded to identify achromatic elements of the root system image. Despite the fact that the tested roots spread widely over an image, they occupy only few percent of its whole area, as shown in Fig. 4 The intensity histogram of $\mathcal{I}_{C}$, theoretically bimodal, can have a very small or even invisible peak associated with root intensities (Fig. 5). Roots chroma can change for semi-transparency of their some parts leading to pastel background colouration of many root pixels. This often makes global roots thresholding ill-conditioned, and local thresholding methods seem to be more appropriate for the image $\mathcal{I}_{C}$. Chroma images usually have some broadband noise, which can be suppressed by averaging each pixel value in its $3 \times 3$ neighbourhood. Highly chromatic discolouration areas like these in Fig. 1 or 2 if present, also have different intensities and should be locally detected in the images $\mathcal{I}_{C_{1}},\left(\mathcal{I}_{C_{2}}\right)$. The authors

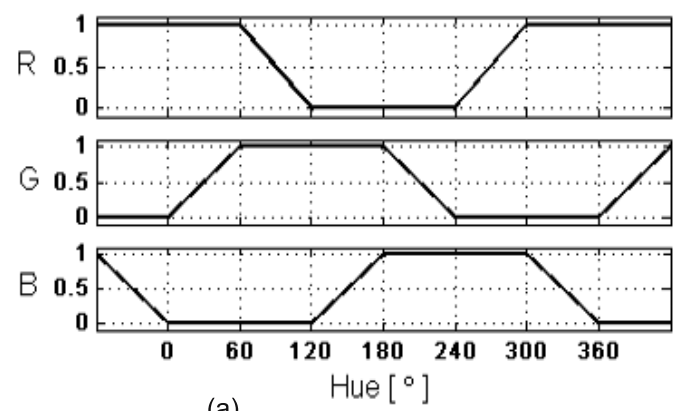

(a)

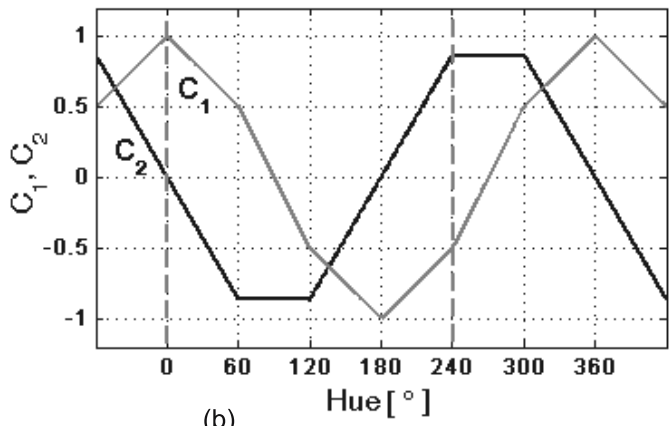

Fig. 3. (a) Relationship between RGB coordinates and hue values, (b) plot of the $C_{1}, C_{2}$ chroma components along the hue axis.

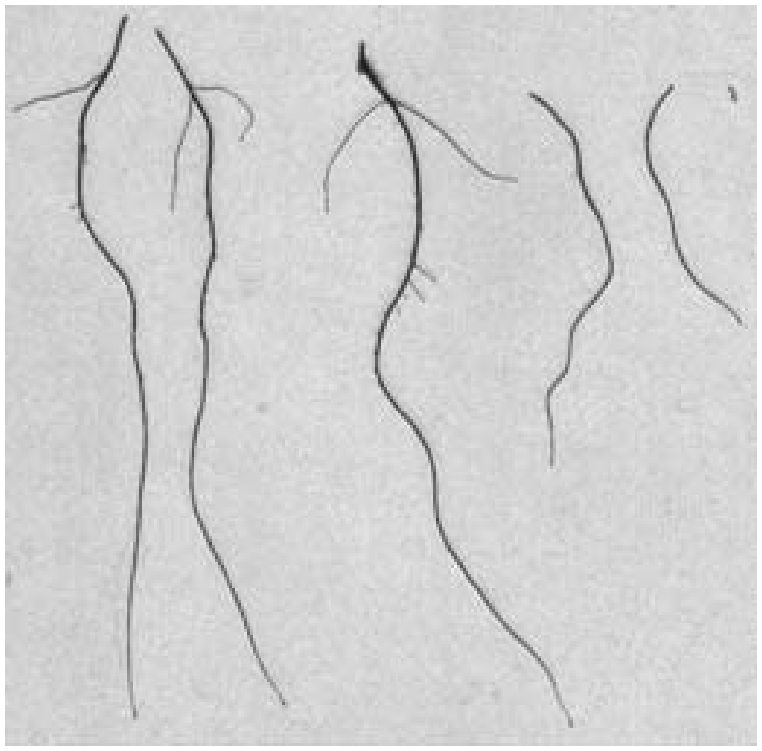

Fig. 4. Scanned chroma image $\mathcal{I}_{C}$ with the set of healthy roots split from one seedling.

applied the relatively fast and efficient Bernsen method (Bernsen, 1986) in a square window $W$ with local contrast limit $\Delta C$.

$$
\begin{array}{r}
t_{B}(W)=\frac{\mathcal{I}_{C m}(W)+\mathcal{I}_{C M}(W)}{2}, \\
\mathcal{I}_{C M}(W)-\mathcal{I}_{C m}(W) \geq \Delta C,
\end{array}
$$




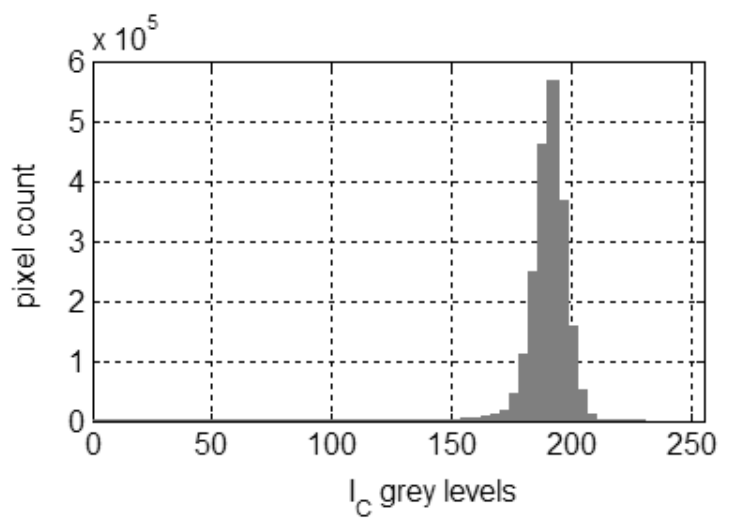

Fig. 5. Grey level histogram of the chroma image $\mathcal{I}_{C}$ from Fig. 4

where $\mathcal{I}_{C m}(W)$ and $\mathcal{I}_{C M}(W)$ denote respectively minimum and maximum brightness of the $\mathcal{I}_{C}$ chroma image in the window $\mathrm{W}$. The image of local threshold values $t_{B}(W)$ was smoothed with bilinear interpolation. When the condition in Eqn. (7) is not fulfilled, the value of the global Otsu threshold for $\mathcal{I}_{C}$ is assigned to the window W (Otsu, 1979). The root system mask binary image $\mathcal{I}_{M}$ is finally evaluated as a logical sum of thresholded inversion of the chroma image $\sim \mathcal{I}_{C}$ and either the red $\mathcal{I}_{C_{1}}$ or the blue $\mathcal{I}_{C_{2}}$ chroma component of $Y C_{1} C_{2}$ :

$$
\mathcal{I}_{M}=\mathbb{T}_{B}\left(\sim \mathcal{I}_{C}\right) \cup \mathbb{T}_{B}\left(\mathcal{I}_{C_{1}}\right)
$$

or

$$
\mathcal{I}_{M}=\mathbb{T}_{B}\left(\sim \mathcal{I}_{C}\right) \cup \mathbb{T}_{B}\left(\mathcal{I}_{C_{2}}\right)
$$

The image processing steps described in Sections 3.1 and 3.2 are depicted in Fig. 6 as a flow diagram. The steps provide only the extraction of the root system mask. Further segmentation is necessary when root dark discolouration regions are to be detected.

\subsection{Root mask image thinning and skeleton sam-} pling. In the current subsection and further on, the authors develop a new approach to the detection of dark discolouration regions in wheat roots with the use of thinning and local fuzzy c-means. It comes out of the observations given below. Nonuniform chroma (Fig. 4) and intensity along the roots suggest that dark type discolourations should be detected as local intensity suppressions in relation to their neighbourhoods. A sequence of square windows around selected sample points has been proposed as local neighbourhoods in the segmentation process. Lengthy shapes, like roots in the binary image $\mathcal{I}_{M}$, can be properly represented by their medial axes (skeleton lines). A satisfactory skeletoning result has been obtained for a series of these images using an algorithm of morphological thinning briefly presented below (Lam et al., 1992; The Mathworks Inc., 2008a). Let $X_{H}(p)$

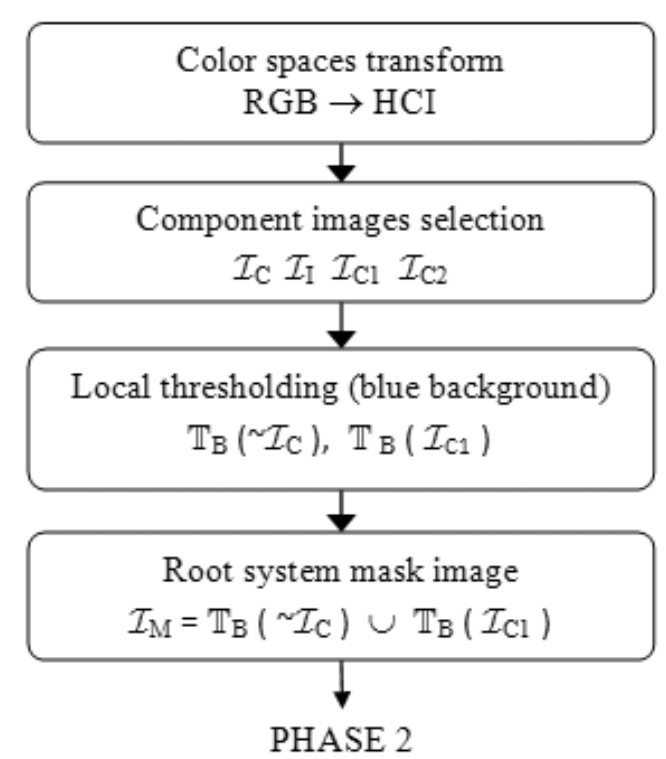

Fig. 6. Flow diagram with the first steps of the algorithm providing the extraction of a root system binary image. $\mathbb{T}_{B}$-Bernsen thresholding with bilinear interpolation.

be the Hilditch crossing number in an 8-neighbourhood $N(p)$ of the root black pixel $p . X_{H}(p)$ represents the number of times one crosses over from a white pixel to a black pixel when the neighbour pixels $N(p)$ are traversed in order, cutting the corners between 8-adjacent black 4neighbours. We have

$$
X_{H}(p)=\sum_{i=1}^{4} b_{i}
$$

where

$$
b_{i}= \begin{cases}1 & \text { if } x_{2 i-1}=0 \wedge\left(x_{2 i}=1 \vee x_{2 i+1}=1\right) \\ 0 & \text { otherwise }\end{cases}
$$

$x_{1}, x_{2}, \ldots, x_{8}$ mean the values of eight neighbours of the pixel $p$, starting with the east neighbour and numbered in counter-clockwise order. The thinned image is divided, using a checkerboard pattern, in two distinct subfields, which are then processed in two subiterations. The black pixel $p$ is deleted if the conditions C1, C2 and C3' (C3”) are all fulfilled:

C1:

$$
X_{H}(p)=1
$$

C2:

$$
2 \leq \min \left\{n_{1}(p), n_{2}(p)\right\} \leq 3,
$$


where

$$
\begin{aligned}
& n_{1}(p)=\sum_{k=1}^{4} x_{2 k-1} \vee x_{2 k}, \\
& n_{2}(p)=\sum_{k=1}^{4} x_{2 k} \vee x_{2 k+1},
\end{aligned}
$$

represent the number of 4 -adjacent pairs of pixels in $N(p)$ containing one or two black pixels,

C3':

$$
\left(x_{2} \vee x_{3} \vee \bar{x}_{8}\right) \wedge x_{1}=0
$$

for the pixel $p$ of the first subfield in the first subiteration,

C3":

$$
\left(x_{6} \vee x_{7} \vee \bar{x}_{4}\right) \wedge x_{5}=0
$$

for the pixel $p$ of the second subfield in the second subiteration.

The above two subiterations deleting black pixels should be repeated until the image $\mathcal{I}_{M}$ stops changing. This thinning produces continuous skeleton lines and exhibits relatively low sensitivity to irregularities in boundaries, which can generate spurious skeleton branches. Skeleton axes $S K$ of a root system form graph like structures. They contain ending and internal nodes connected by branches as sequences of consecutive image pixels between the nodes:

$$
\begin{aligned}
& S K=\left\{B_{i}: i=1, \ldots, N_{B}\right\}, \\
& B_{i}=\left\{\left(x_{j}, y_{j}\right): j=1, \ldots, N_{i}\right\},
\end{aligned}
$$

where $B_{i}$ is the $i$-th skeleton branch with $N_{i}$ pixels length. Every skeleton pixel is analysed in a $3 \times 3$ neighbourhood (Hasthorpe and Mount, 2007).

Skeleton analysis comprises three steps:

Step 1. Identification of end nodes and internal nodes.

Step 2. Tracking and storing all branches between nodes.

Step 3. Sampling every branch with a step $\Delta l$ of constant length along the tracking path.

The authors developed their own method of tracking root skeleton branches, detecting and accepting possible root loops. Given Euclidean distances of all branch pixels $\left(x_{j}, y_{j}\right)$ to their predecessors (neighbours) as

$$
l(j)= \begin{cases}1 & \text { for the orthogonal neighbour, } \\ \sqrt{2} & \text { otherwise }\end{cases}
$$

and the branch cumulative length function

$$
L(1)=0, \quad L(j)=L(j-1)+l(j),
$$

where $j=2, \ldots, N_{i}$, sample pixels $S(x, y)$ are determined by the constant, predefined increments $\Delta l$ of the $L\left(x_{j}, y_{j}\right)$ function values (Fig.7).

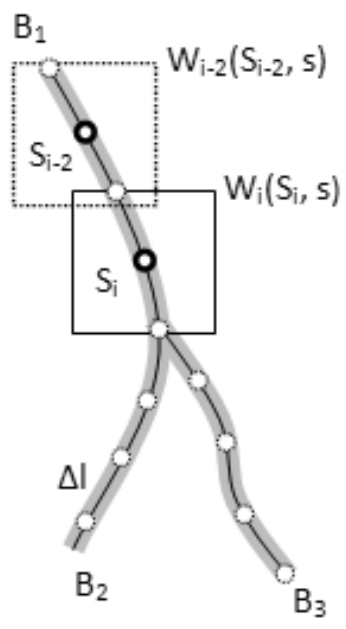

Fig. 7. Illustrative fragment of a root system with skeleton branches $B_{i}$ (black medial axes), sample points $S_{i}$ and segmentation square windows $W_{i}$ with side $s$.

3.4. Local fuzzy c-means segmentation. Dark type discolourations are featured by lower intensities than their neighbourhoods in the image $\mathcal{I}_{I}$. Problems in the extraction of these regions include the ambiguity of their exact locations due to varying brightness of neighbouring pixels in healthy tissue, a relatively small size, unsharp borders and the presence of noise. For image segmentation uncertainty problems, a suitable tool can be the Fuzzy C-Means method (FCM) applying fuzzy set theory (Bezdek, 1981; Horvath, 2006; Mohamed et al., 1999; The Mathworks Inc., 2008b). The method is an iterative procedure which minimizes membership uncertainty of feature space data to a predefined number of clusters. It is realized by minimizing the objective function

$$
J_{m}=\sum_{i=1}^{N} \sum_{j=1}^{M} u_{i j}^{m}\left\|d_{i}-v_{j}\right\|^{2}
$$

where $m \geqslant 1$ is the "fuzzification" parameter, $u_{i j}$ is the degree of membership of the $d_{i}$ data in the cluster $j, v_{j}$ is the cluster centre and $\|\cdot\|$ is any norm expressing the distance between the measured data and the cluster centre. The case of the Euclidean distance and two cluster centres of dark and bright pixels $(M=2)$ with intensity values $D_{i}=\left[d_{i}\right], i \in\{1,2, \ldots, N\}$ as input data is taken into account. At the $k$-th iteration step, the membership values $u_{i j}$ and the cluster centres $v_{j}$ are updated as follows:

- calculate the new centre vectors $V^{(k)}=\left[v_{j}\right]$ with $U^{(k-1)}=\left[u_{i j}\right]$,

$$
v_{j}=\frac{\sum_{i=1}^{N} u_{i j}^{m} \cdot d_{i}}{\sum_{i=1}^{N} u_{i j}^{m}}
$$




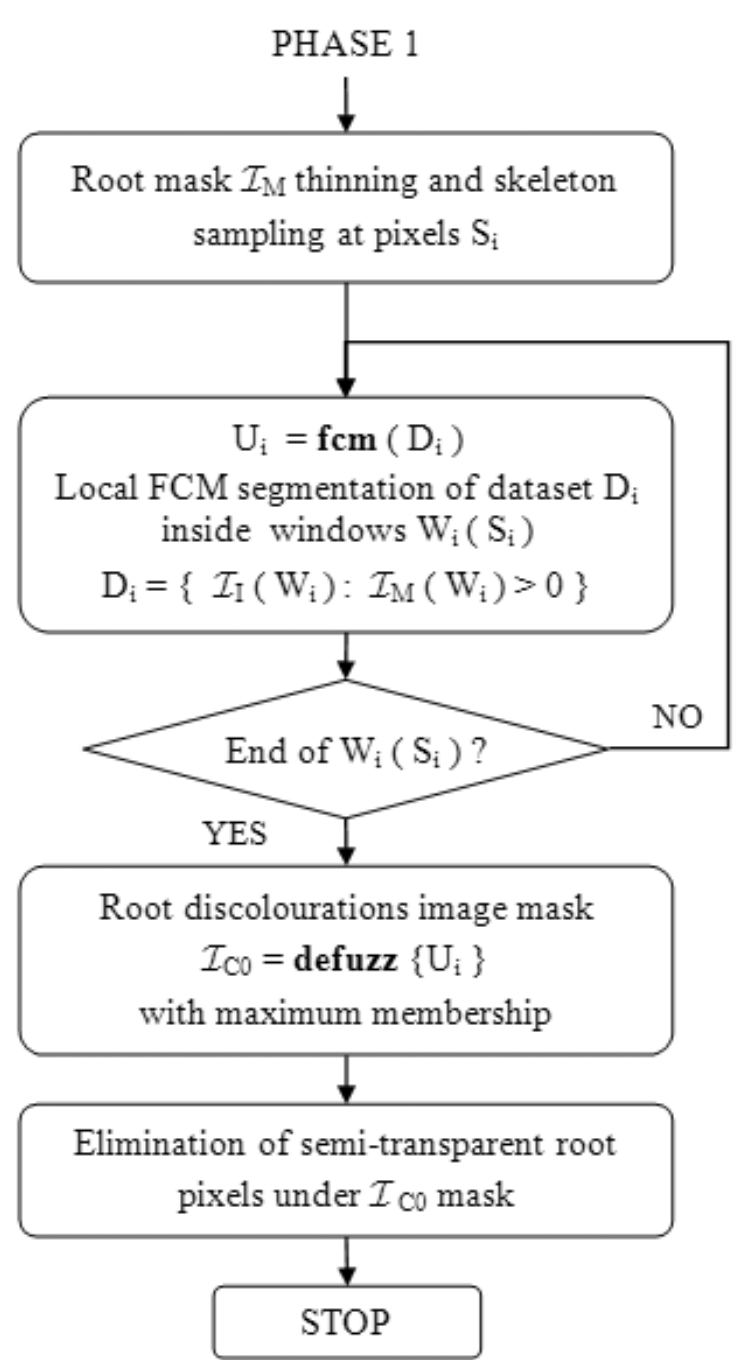

Fig. 8. Flow diagram with the final steps of the algorithm accomplishing the detection of dark discolourations.

- update $U^{(k)}$ with the new $V^{(k)}$,

$$
u_{i j}=\frac{1}{\sum_{k=1}^{M}\left(\frac{\left\|d_{i}-v_{j}\right\|}{\left\|d_{i}-v_{k}\right\|}\right)^{2 /(m-1)} .}
$$

This iteration will stop at the $k$-th step when

$$
\left\|U^{(k)}-U^{(k-1)}\right\|<\varepsilon \text { or } k>k_{\max },
$$

where $\varepsilon, k_{\max }$ mean termination criteria. The FCM method converges only to a local minimum or a saddle point of $J_{m}$. To cluster the image $\mathcal{I}_{I}$ data of the roots $\left(\mathcal{I}_{M}>0\right)$ into groups of bright or dark pixels, the authors applied the fuzzy c-means clustering described by Eqns. (21)-23) to each window $W_{i}$ centred at the skeleton sample point $S_{i}$ (Fig.77):

$$
V_{i}, U_{i}, J_{m i} \leftarrow F C M\left(D_{i}, M, m, \varepsilon, k_{\max }\right),
$$

where

$$
D_{i}=\left\{\mathcal{I}_{I}\left(W_{i}\right): \mathcal{I}_{M}\left(W_{i}\right)>0\right\},
$$

$D_{i}$ is the dataset of image intensity values in a selected region, $M=2$ is the number of clusters, $m=2$ is the "fuzzification" parameter, $\varepsilon$ and $k_{\max }$ stand for iterations stopping criteria, $V_{i}$ is the array of final cluster centres, $U_{i}$ is the final membership array, $J_{m i}$ denotes values of the objective function during iterations. For every window $W_{i}$, the membership dataset $U_{i}$ obtained in Eqn. (24) was mapped into the image raster domain $\mathcal{I}_{M}^{-1}=X \times Y$ as the array $P_{i}$ (Eqn. (26)).

$$
\forall_{i}\left\{U_{i}, x_{i}, y_{i}\right\} \rightarrow\left(P_{y x}\right)_{i} .
$$

The local membership values $\left(p_{y x}\right)_{i}$, for each pixel $(x, y)$, descended from overlapping windows $W_{i}$ should be averaged:

$$
p_{y x}=\frac{1}{n_{y x}} \sum_{i=1}^{n_{y x}}\left(p_{y x}\right)_{i}, \quad \forall n_{y x}>0,
$$

where $n_{y x}$ is an entry of the two-dimensional $N_{y x}$ array with the $\mathcal{I}_{M}^{-1}$ domain and represents the count of windows $W_{i}$ overlapping in the pixel $(x, y)$. The entries $p_{y x}$ of the array $P_{y x}$ are pairs of calculated clusters membership values completing to one. During programming in MATLAB, environment partial cluster memberships and their counts are cumulated only in two arrays: $P_{y x}(Y \times X \times 2)$ and $N_{y x}(Y \times X)$, stored as sparse types. Calculations of local membership sets $U_{i}$ in windows $W_{i}$ (Eqn. (24) can give inappropriate segmentation results in the following cases:

- centres of the two expected clusters are too close in the feature space:

$$
\left\|V_{i, 1}-V_{i, 2}\right\|<\varrho_{\min },
$$

- the number of pixels in the dataset $D_{i}$ (Eqn. (25) is too small to get reliable membership values $U_{j}$ (Eqn. (24)):

$$
\left|D_{i}\right|<|D|_{\min }
$$

In these situations, local arrays $U_{i}$ for the sets of points $\left\{x_{i}, y_{i}\right\}$ must be substituted with equivalent values of the global membership array $U$ created through global FCM clustering (Eqn. (21)-(23)), symbolically described by

$$
\begin{aligned}
& V, U, J_{m} \leftarrow F C M\left(D_{M}, M, m, \varepsilon, k_{\max }\right), \\
& D_{M}=\left\{\mathcal{I}_{I}: \mathcal{I}_{M}>0\right\}
\end{aligned}
$$

The employed defuzzification procedure of the fuzzy partition array $P_{y x}=\left[P_{y x}(0), P_{y x}(1)\right]$ assigns every image pixel $(x, y)$ inside the root mask $\left(\mathcal{I}_{M}>0\right)$ to class 
$\mathcal{C}_{k \max }$ with the highest membership:

$$
\begin{aligned}
& \mathcal{C}_{k \max }=\arg \left\{\max _{k} P_{y x}(k)\right\}, \\
& k=0,1, \quad(x, y) \in D_{M}^{-1} .
\end{aligned}
$$

Let $\mathcal{C}_{1}$ be the class of bright root pixels and $\mathcal{C}_{0}$ the class of dark pixels in the segmentation region. The binary result of the proposed FCM segmentation is the image

$$
\mathcal{I}_{\mathcal{C}_{0}}= \begin{cases}1 & \text { if } \mathcal{C}_{k \max }=\mathcal{C}_{0} \\ 0 & \text { otherwise }\end{cases}
$$

3.5. Elimination of semi-transparent regions and morphological postprocessing. Some pixels of root objects can be misclassified in Eqn. (34) as discolourations relaying only on the values of the intensity image $\mathcal{I}_{I}$ under the root mask $\mathcal{I}_{M}>0$. Lower intensities of these pixels come from little background colouration (e.g., blue) through semi-transparency. This phenomenon is eliminated, as shown in

$$
\mathcal{I}_{\mathcal{C}_{0}}^{\prime}= \begin{cases}1 & \text { if } \mathcal{I}_{\mathcal{C}_{0}}=1 \text { and } \mathcal{I}_{S T}=0 \\ 0 & \text { otherwise }\end{cases}
$$

by the image mask $\mathcal{I}_{S T}$ of partially transparent pixels

$$
\mathcal{I}_{S T}= \begin{cases}1 & \text { if } \mathcal{I}_{B}>\max \left(\mathcal{I}_{R}, \mathcal{I}_{G}\right), \\ 0 & \text { otherwise. }\end{cases}
$$

A second phase of the proposed algorithm described in Sections 3.3, 3.4, 3.5 is shown as a flowchart in Fig. 8. Binary discolouration regions are finally processed with area identification and morphological functions from MATLAB libraries. The binary image $\mathcal{I}_{\mathcal{C}_{0}}$ can be considered as a set of objects (regions) $\{O\}$ of 8 -adjacent pixels with nonzero values on a zero background. All these objects, identified by numerical labels, are featured by the area properties $A_{O}$ defined as numbers of the pixels. Objects with small areas, below a certain limit value $A_{\text {min }}$, are considered as spurious data and eliminated.

$$
(x, y) \in O \wedge A_{O}<A_{\min } \Rightarrow \mathcal{I}_{\mathcal{C}_{0}}(x, y)=0 .
$$

The remaining, greater objects are then smoothed by morphological opening with the structuring element $D_{R}$ of disk type with radius $R$ (Serra, 1986; The Mathworks Inc., 2008a):

$$
\mathcal{I}_{\mathcal{C}_{0}}^{\prime}=\gamma_{D_{R}}\left(\mathcal{I}_{\mathcal{C}_{0}}\right)
$$

\section{Experimental results and conclusions}

The proposed segmentation method was applied to the population of several images of wheat root systems prepared as described in Section 2, The acquired images

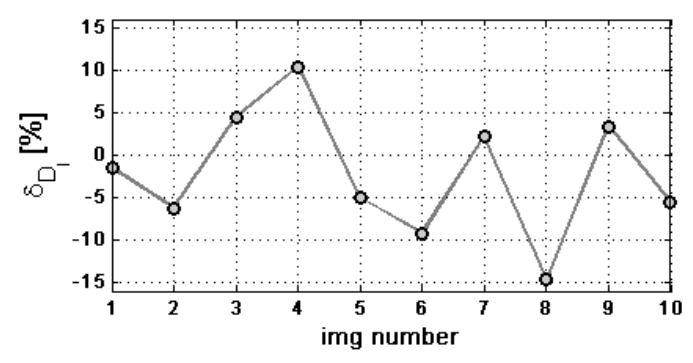

Fig. 11. Discolouration index errors $\delta_{D_{I}}$ of the automatic segmentation method in comparison with the manual method, for the set of tested images.

included well-developed normal roots (Fig. 4), as well as those heavy metal-treated, topologically reduced systems with dark type discolourations (Fig. 9a)). In the first case, the segmentation process only extracts roots from the background, in the second-it should also separate dark regions of roots. Figure 9 a) shows in greys an original colour image $\mathcal{I}_{R G B}$ of the root system, where the background is blue and the roots are white with dark spots (grey-brownish). Colour space transformation (Eqns. (1)-(5) produces the chroma image $\mathcal{I}_{C}$ visible in Fig. 9b). From Figs. 9(c) and (d) we can see that the global Otsu thresholding of $\mathcal{I}_{C}$ can "loose" some parts of root masks compared with local thresholding using the Bernsen method (Eqn. (6)). The local window $W[31 \times 31]$ and the local contrast limit $\Delta C=15$ in the range $[0,255]$ (Eqn. (7)) were assumed as algorithm parameters. The discolouration regions depicted in Fig. 10 (c) were detected by global histogram thresholding of the intensity image $\mathcal{I}_{I}$ within root masks. They differ from the results of the proposed method shown in Fig. 9f).

During the execution of local FCM, the following parameter values were assumed: the minimization progress $\varepsilon=1 \mathrm{e}-2$, the maximum iterations $k_{\max }=150$, the local window $W_{i}$ with side $s=65$, the skeleton sampling step $\Delta l=32$ (Fig. 7). Comparing the results in Fig. 9 (f) and Fig. 10 (c) with the original example image (Fig. 9(a)), it can be easily noticed that the global method drops some discolourations and over-extends the others. Standard, hand-labelled segmentations were carried out for the same series of images as in the automatic method.

Assume the goal of our segmentation to be the estimation of the following discolouration index:

$$
D_{I}=\frac{A_{D}}{A_{R}},
$$

where $A_{R}$ is the area of the roots mask in the segmented image, $A_{D}$ is the area of discolourations inside the root mask. It is supposed that an average value of this index, in the set of root images, is related to a certain dose of contamination by metal compounds (e.g., $\mathrm{NiSO}_{4}$ ). Then the 


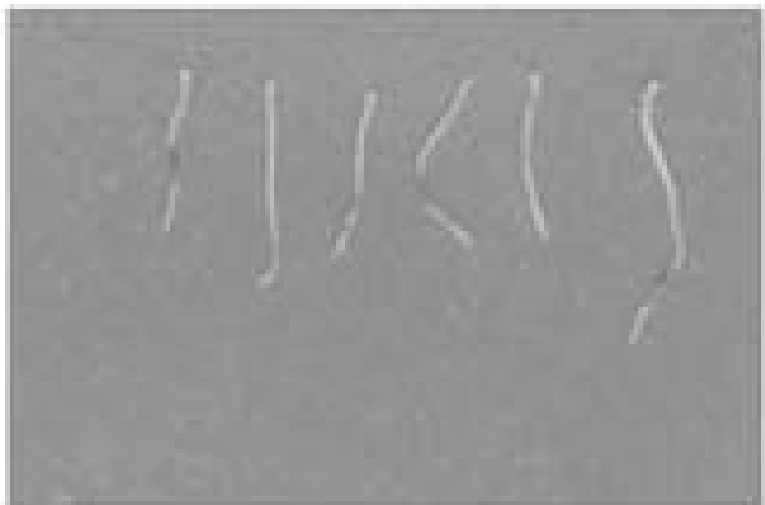

(a)

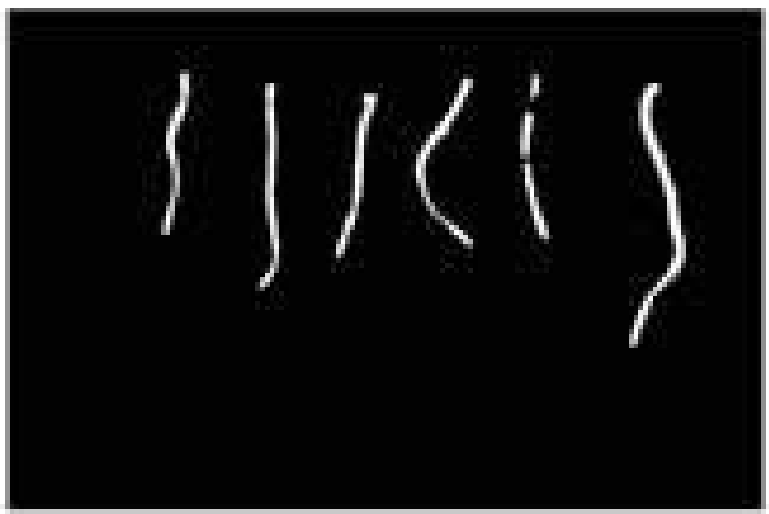

(c)

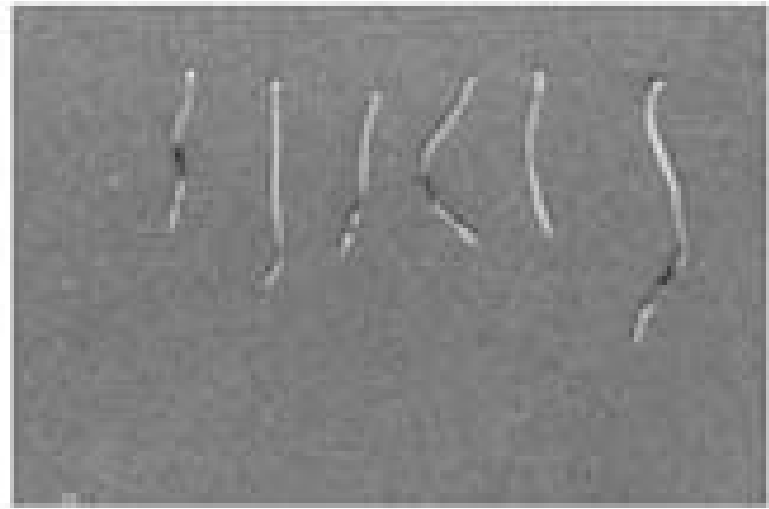

(e)

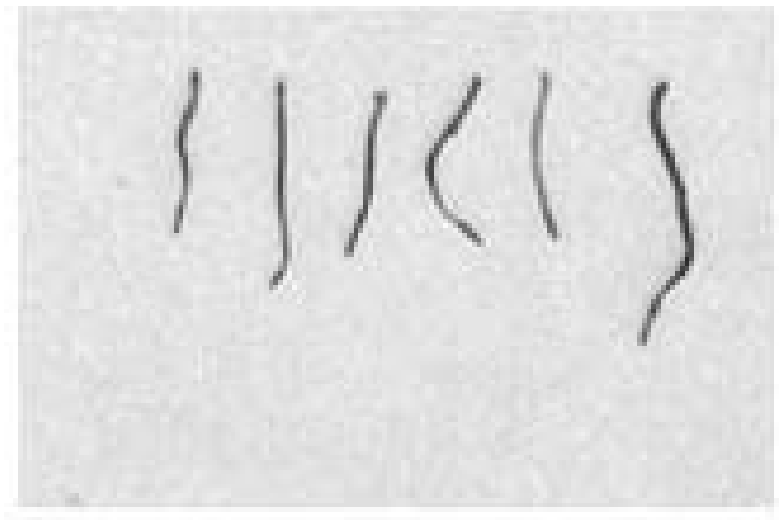

(b)

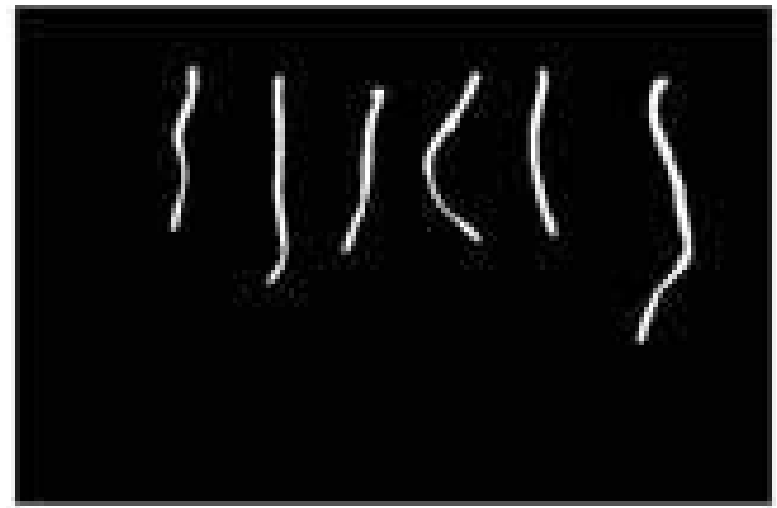

(d)

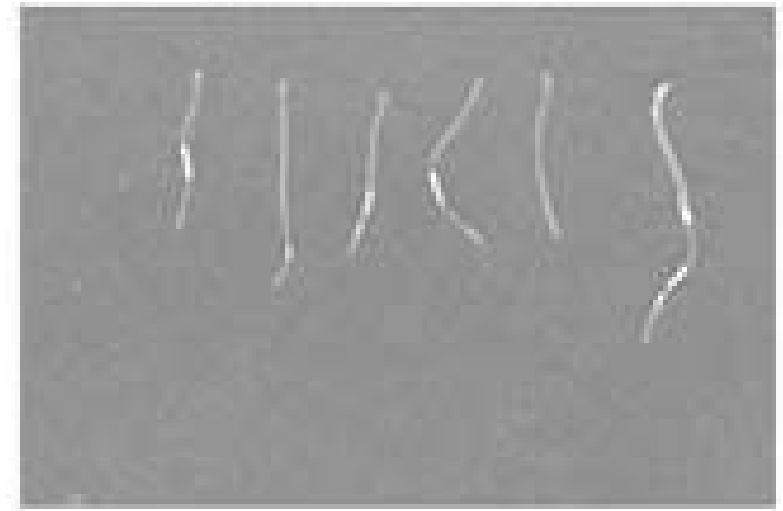

(f)

Fig. 9. Example stages of root system image processing: (a) original image $\mathcal{I}_{R G B}$ of Ni-treated roots on a blue background, (b) chroma image $\mathcal{I}_{C}$, (c) binary root mask image after the global Otsu thresholding, (d) binary root mask image $\mathcal{I}_{M}$ after local thresholding, (e) intensity image $\mathcal{I}_{I}$ from the HCI space corresponding with (a), (f) results of discolourations detection with our method-marked white regions overlapped on $\mathcal{I}_{R G B}$.

quality of segmentation with the proposed image processing method can be expressed by the relative error:

$$
\delta_{D_{I}}=\frac{D_{I}^{I}-D_{I}^{M}}{D_{I}^{M}},
$$

where

$$
D_{I}^{I}=\frac{A_{D}^{I}}{A_{R}^{I}}, \quad D_{I}^{M}=\frac{A_{D}^{M}}{A_{R}^{M}} .
$$

The symbols $A_{D}^{I}\left(D_{I}^{I}\right)$ and $A_{D}^{M}\left(D_{I}^{M}\right)$ mean areas (discolouration indexes) found by image processing and 


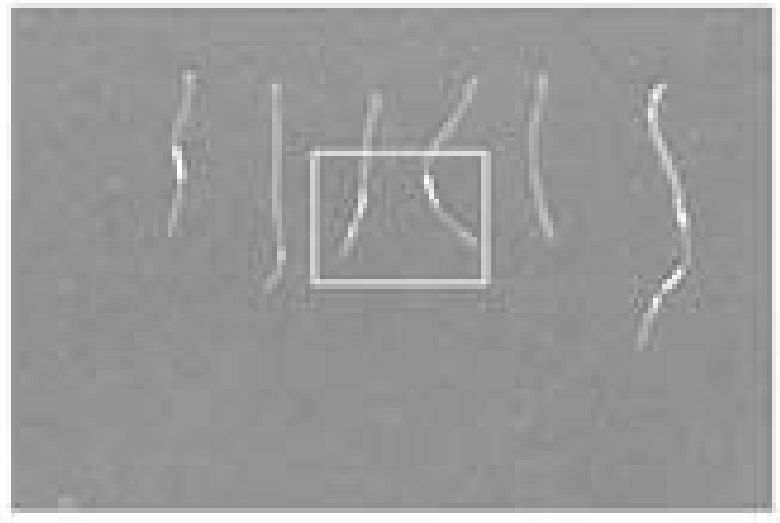

(a)

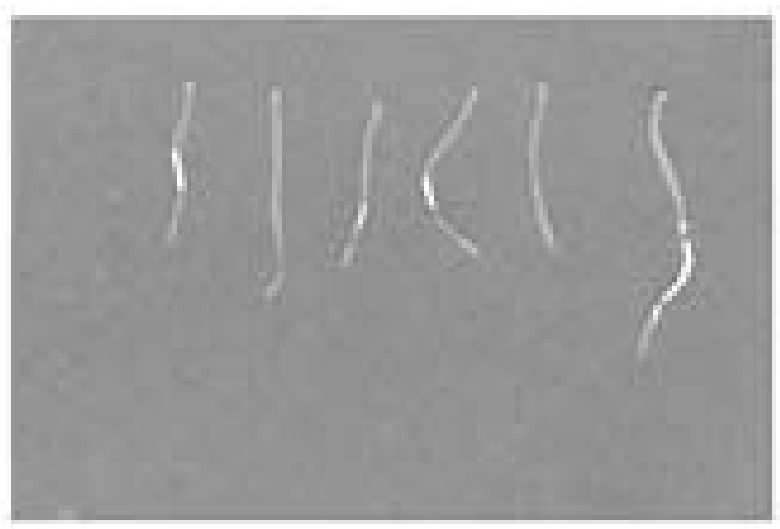

(c)

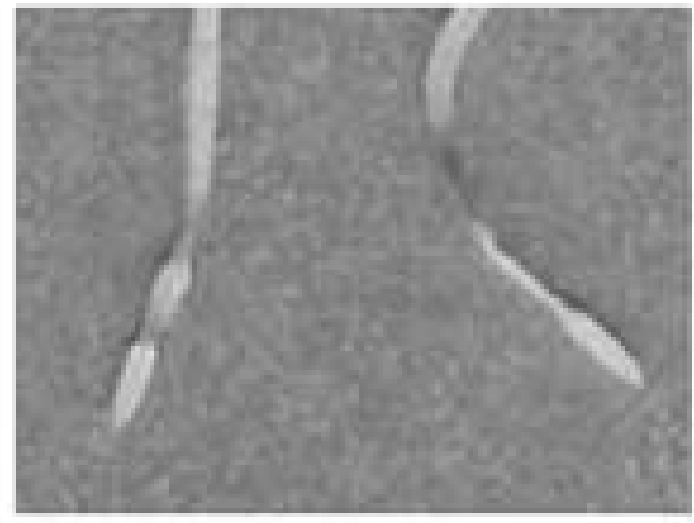

(b)

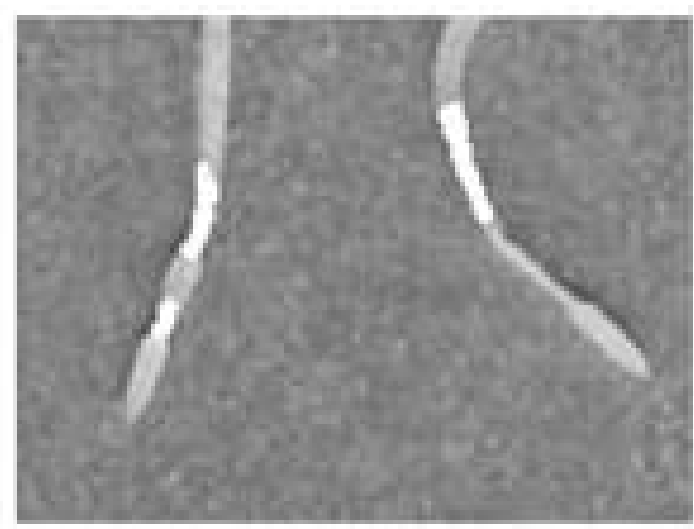

(d)

Fig. 10. Example stages of image processing: (a) discolourations detection results for the proposed method with a zooming rectangle, (b), (d) contents of the zoomed window shown in (a) for $\mathcal{I}_{R G B}$ without and with overlapped discolourations, (c) white marked discolourations from global thresholding of $\mathcal{I}_{I}$ under the $\mathcal{I}_{M}$ mask.

visual inspection methods, respectively. Similarly, $A_{R}^{M}$ and $A_{R}^{I}$ represent areas of the root mask estimated manually and by image segmentation. All of the areas are calculated as the numbers of image pixels. The data evaluated manually are regarded as true, reference values. Figure 11 shows discolouration index errors for a series of $N=10$ images. An average error in this set marked as $\bar{\delta}_{D_{I}}$ is equal $-2.15 \%$ and its standard deviation is $7.4 \%$.

The most important parameters assumed in the algorithm are as follows:

- the size of the Bernsen local window $W_{B}\left[s_{B} \times s_{B}\right]$ and the local contrast limit $\Delta C$ within this window,

- the size of the local FCM window $W_{i}\left(S_{i}, s\right)$ and the root image skeleton sample distance $\Delta l$,

- local FCM algorithm stopping criteria: the minimization progress $\varepsilon$ and the maximum iteration limit $k_{\max }$,
- minimum distance $\varrho_{\text {min }}$ between two cluster centres in local FCM (Eqn. (28) and the minimal number of the local pixels considered $\left|D_{\min }\right|$ (Eqn. (29)),

- the discolouration areas lower limit $A_{\min }=64$ in Eqn. (37) and the morphological opening radius of the structuring element $R=1$ (Eqn. (38)).

The size $s_{B}=31$ of the local window has been selected experimentally. It is small enough to get a proper threshold for local chroma values of roots in the image $\mathcal{I}_{C}$. Resizing the window between $15 \times 15$ and $65 \times 65$ pixels results in a change of the average error: $\bar{\delta}_{D_{I}} \in$ $[-1.8 \%, 4.2 \%]$. Local contrast limit $\Delta C=15$ in the range $[0,255]$ for the Bernsen method (Eqn. (7)) is mainly determined by the noise level in the image $\mathcal{I}_{C}$. To lower this limit, the image is averaged a little before thresholding in the $3 \times 3$ neighbourhood around each pixel. Changing the local FCM window size from $s=33$ to $s=97$ by $\Delta s=8$ (in proportion to the skeleton sample distance) gives the average error $\bar{\delta}_{D_{I}}$ fluctuating in a range 
Table 1. Comparison of image sizes, roots features and execution times for the proposed method.

\begin{tabular}{|c|cc|cc|}
\hline $\begin{array}{c}\text { image } \\
\text { size } \\
{[\mathrm{px} \times \mathrm{px}]}\end{array}$ & \multicolumn{2}{|c|}{ roots } & \multicolumn{2}{c|}{ execution time } \\
length & area & phase 1 & phase 2 \\
{$\left[\mathrm{px}^{2}\right]$} & {$[\mathrm{s}]$} & {$[\mathrm{s}]$} \\
\hline \hline $1470 \times 1440$ & 6682 & 38004 & 2.92 & 2.46 \\
$690 \times 460$ & 1065 & 7159 & 0.48 & 0.34 \\
$1380 \times 920$ & 1065 & 7159 & 1.66 & 0.75 \\
\hline
\end{tabular}

$[-15.2 \%, 10.3 \%]$. Varying $\varepsilon$ from $1 e-3$ to $1 e-1$ with proposed $k_{\max }$ has no visible influence on discolouration regions in the tested set of images. The changes in the cluster distance $\varrho_{\min }$ (Eqn. 28) from 8 to 20 in the range of $[0,225]$ practically do not affect discolouration region areas. The average error $\bar{\delta}_{D_{I}}$ increases to about $10 \%$ for $\varrho_{\min }=32$. The value $\left|D_{\min }\right|$ is assumed typically as a product of half FCM window side and average root thickness. Its variations $\mp 50 \%$, for the fixed FCM window size $65 \times 65$ pixels, do not have significant influence on the index $D_{I}$. Assigning no limit of the lowest discolouration areas $\left(A_{\min }=0\right)$ and no morphological opening $(R=0)$ in Eqns. (37) and (38) increases the average relative error to $\bar{\delta}_{D_{I}}=3.4 \%$.

The method was executed in MATLAB 2008 environment using a $\mathrm{PC}$ with dual core processor Intel Core (TM)2 Duo T5750 $2 \mathrm{GHz}$, 4 GB RAM and operating system Windows Vista Home Premium. Example execution times are presented in Table 1. Data in the first row are related to the image of healthy roots in Fig. 4. The second and third rows include processing parameters of the same roots from Fig. 9a), but for different image margins. In the proposed method, execution time of the first phase depends on the area of the processed image $\mathcal{I}_{C}$. Colour space transformations, filtering and local thresholding refer to each image pixel. The same is with the second phase regarding morphological and filtering operations. Therefore, before processing, scanned images should be limited to their rectangular regions including visible roots plus reasonable margins. The execution time of local FCM procedure and semi-transparency elimination during second phase depends on the number of pixels inside the roots mask $\mathcal{I}_{M}$. The total length of roots determines the times of skeleton tracking and sampling. Fortunately, contaminated root systems with discolourations are significantly reduced in area and length. The second phase of the algorithm is normally unused for images with healthy roots like this one shown in Fig. 4 and described in the first row of Table 1 In the future it is planned to study biochemical reactions of wheat seedlings to heavy metal stress. The statistical dependency will be searched between the data obtained by image analysis and those acquired by biochemical analysis. This could make possible to create an estimator of chemical pollution level of plants by means of image processing instead of long-lasting and expensive biochemical studies.

\section{References}

Bernsen, J.(1986). Dynamic thresholding of grey-level images, Proceedings of the 8th International Conference on Pattern Recognition, Paris, France, pp. 1251-1255.

Bezdek, J. C. (1981). Pattern Recognition with Fuzzy Objective Function Algorithms, Plenum Press, New York, NY.

Glasbey, C. A. and Horgan, G. W. (1995). Image Analysis for the Biological Sciences, John Wiley \& Sons, Inc., New York, NY.

Gonzalez, E. R., Woods, R. E. and Eddins S. L. (2004). Digital Image Processing Using MATLAB, Prentice Hall, Upper Saddle River, NJ.

Gonzalez, R. C. and Woods, R. C. (2008). Digital Image Processing, Prentice Hall, Upper Saddle River, NJ.

Hasthorpe, J, and Mount, N. (2007). The generation of river channel skeletons from binary images using raster thinning algorithms, http://ncg.nuim.ie/gisruk/ materials/proceedings/PDF/P7.pdf.

Horvath, J. (2006). Image segmentation using fuzzy c-means, http://bmf.hu/conferences/sami2006/ JurajHorvath.pdf.

Jung, V., Olsson, E., Caspersen, S., Asp, H., Jensen, P. and Alsanius, B. W. (2004). Response of young hydroponically grown tomato plants to phenolic acids, Scientia Horticulturae 100(1-4): 23-37.

Lam, L., Lee, S-W. and Suen, C. Y. (1992). Thinning methodologies-A comprehensive survey, IEEE Transactions on Pattern Analysis and Machine Intelligence 14(9): 869-885.

Lambert, P. and Carron, T. (1999). Symbolic fusion of luminance-hue-chroma features for region segmentation, Pattern Recognition 32(11): 1857-1872.

Mohamed, N. A., Ahmed, M. N. and Farag A. (1999). Modified fuzzy c-means in medical image segmentation, Proceeding of the IEEE International Conference on Acoustics, Speech, and Signal Processing, Phoenix, AZ, USA, Vol. 6, pp. 3429-3432.

Nord, E. (2008). Other Uses for WinRhizo, http://roots . psu.edu/en/node/36.

Otsu, N. (1979). A threshold selection method from grey-level histograms, IEEE Transactions on Systems, Man, and Cybernetics 9(1): 62-66.

Regent Instruments Inc. (2008). WinRHIZO For root morphology and architecture measurement, http://www.regent.qc.ca/products/rhizo/ Rhizo.html.

Seregin, I. V. and Kozhevnikova, A. D. (2009). Enhancement of nickel and lead accumulation and their toxic growthinhibitory effects on amaranth seedlings in the presence of calcium, Russian Journal of Plant Physiology 56(1): 8084. 
Serra, J., (1986). Introduction to mathematical morphology, Computer Vision, Graphics and Image Processing 35(3): 283-305.

Smit, L. A., Bengough, A. G.,Engels, C., Van Noordwijk, M., Pellerin, S. and Van de Geijn, S. C. (2000). Root Methods: A Handbook, Springer Verlag, Heidelberg.

Terry N., Zayed A.M., de Souza M.P. and Tarun A.S. (2000). Selenium in higher plants, Annual Review of Plant Physiology and Plant Molecular Biology 51: 401-432.

The Mathworks Inc. (2008a). Image Processing Toolbox User's Guide, http: //www.mathworks.com/products / matlab/.

The Mathworks Inc. (2008b). Fuzzy Logic Toolbox User's Guide, http: / /www.mathworks. com/products / matlab/.

Vincent, L. and Soille, P. (1991). Watersheds in digital spaces: An efficient algorithm based on immersion simulations, IEEE Transactions on Pattern Analysis and Machine Intelligence 13(6): 583-598.

Zobel, R. W., Kinraide, T. B. and Baligar, V.C. (2007). Fine root diameters can change in response to changes in nutrient concentration, Plant and Soil 297(1-2): 243-254.

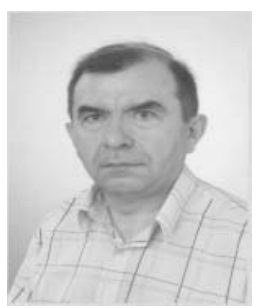

Jarosław Gocławski received the M.S.E. degree in electronics in 1977 and then the Ph.D. degree in 1986, both from the Electrical Faculty of the Technical University of Łódź, Poland. From 1977 to 1989 he worked on image processing, analysis and tomographic reconstructions as an academic teacher at the Institute of Electronics of the same university. From 1989 to 2005 he was employed as a chief of the Informatics Department at IMAL Ltd., in Łódź, where he worked on the design of specialized software and hardware image processing solutions for medicine and biology. Since 2006 he has been a lecturer at the Computer Engineering Department of the Technical University of Łódź. His research interests include image processing and analysis for the purpose of measurements in textile industry and the biology of plants. In the last three years he has co-authored several journal and conference papers in these areas.

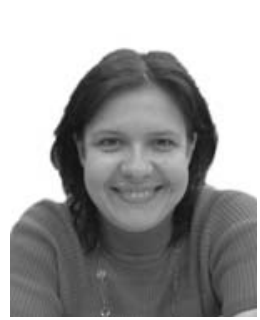

Joanna Sekulska-Nalewajko received the M.S. degree in environmental biology in 1996 and then the Ph.D. degree in phycology and hydrobiology in 2001, all at the Faculty of Biology and Environmental Protection, University of Łódź, Poland. In 2001 she was employed at the Computer Engineering Department of the Technical University of Łódź. Since 2005 she has been an assistant professor. She works on diatom ecology in modern and past inland water environments. Another research interest of hers is concerned with image analysis of plant cells for diagnostic purposes and 3D graphical simulations as well as the modelling of ecological processes during river flooding. She is an author of several papers on this topic.

Ewa Gajewska received the M.S. degree in biology in 1994 and then the Ph.D. degree in biology in 2001, all at the Faculty of Biology and Environmental Protection, University of Łódź, Poland. Since 2001 she has been an assistant professor at the Department of Plant Physiology and Biochemistry of the same university. Her research interest include biochemical response of plants to environmental stress factors, especially excess heavy metal concentrations. She studies the influence of heavy metals on metabolism, pro- and antioxidative reactions of wheat plants.

Marzena Wielanek received the M.S. degree in biology in 1995 and then the Ph.D. degree in biology in 2002, all at the Faculty of Biology and Environmental Protection, University of Łódź, Poland. Since 2002 she has been an assistant professor at the Department of Plant Physiology and Biochemistry of the same university. Her research interest is in the utilization of plant in vitro cultures for the production of bioactive secondary metabolites. At present her studies are focused on the incorporation of selenium into organoselenium compounds by hairy root cultures.

Received: 25 March 2009

Revised: 22 July 2009 